\title{
Síndrome do anticorpo antifosfolípide: estudo comparativo das formas primária e secundária(*)
}

\author{
Antiphospholipid antibody syndrome: \\ a comparative study between primary and secondary forms
}

\author{
Max Victor Carioca Freitas ${ }^{(1)}$, Lucienir Maria da Silva ${ }^{(1)}$, Flavio Calil Petean ${ }^{(1)}$, Ivan Fiore de Carvalho ${ }^{(1)}$, \\ Rendrik França Franco ${ }^{(2)}$, Eduardo Antônio Donadi ${ }^{(1)}$, Paulo Louzada Júnior ${ }^{(1)}$
}

\section{RESUMO}

Objetivo: Traçar um perfil clínico e laboratorial da síndrome do antifosfolípide (SAF), comparando a primária (SAFP) com aquela secundária (SAFS) ao lúpus eritematoso sistêmico (LES). Métodos: Avaliamos 27 pacientes com SAFP e 32 com SAFS ao LES, acompanhados no Ambulatório de Colagenoses do HC/FMRP/ USP, quanto à ocorrência de trombose arterial, venosa, perda gestacional, livedo reticular, fenômeno de Raynaud, anemia hemolítica auto-imune, plaquetopenia, linfopenia, anticorpos anticardiolipina, anticoagulante lúpico, antinucleares, anti-Sm e VDRL. Os anticorpos anticardiolipina e anti-Sm foram pesquisados por ELISA, os antinucleares por imunofluorescência indireta e o anticoagulante lúpico pelo tempo de protrombina diluída, tempo de coagulação do caulin ou tempo do veneno de víbora de Russell diluído. Para análise estatística utilizamos o teste exato de Fisher bicaudal. Resultados: Observamos aumento da freqüência de trombose arterial na SAFP $(59,3 \%$ vs $25,0 \%, p=0,009)$ e de trombose venosa na SAFS $(53,1 \%$ vs $33,3 \%, p>0,05)$, enquanto não houve diferenças entre as freqüências de perda gestacional $(50,0 \%$ vs 56,7\%), fenômeno de Raynaud (18,5\% vs 18,8\%), livedo reticular (18,5\% vs $12,5 \%)$, anticoagulante lúpico $(33,3 \%$ vs $37,5 \%)$ e anticardiolipina $\operatorname{IgG}(79,2 \%$ vs $72,4 \%)$ e $\operatorname{IgM}(58,4 \%$ vs $65,5 \%)$. Ademais, observamos aumento significante de linfopenia $(71,2 \%$ vs $7,4 \%, p<0,0001)$, de anticorpos antinucleares (100\% vs 7,4\%, $p<0,0001)$ e de VDRL positivo $(47,1 \%$ vs $5,0 \%, p=0,005)$ na SAFS ao LES quando comparada com a SAFP. Conclusões: As manifestações clínicas e laboratoriais são semelhantes na SAFP e na SAFS ao LES, sendo a trombose arterial mais comum na SAFP, enquanto a presença de linfopenia, anticorpos antinucleares e VDRL positivo está associada com a SAFS ao LES.

Palavras-chave: síndrome do antifosfolípide, lúpus eritematoso sistêmico, trombose, anticorpo anticardiolipina, anticoagulante lúpico.

\begin{abstract}
Objective: To study clinical and laboratory features of primary antiphospholipid syndrome (PAPS) and that of secondary (SAPS) to systemic lupus erythematosus (SLE). Methods: Twenty-seven PAPS and 32 SAPS patients were investigated in relation to the presence of arterial/venous thrombosis, fetal loss, reticular livedo, Raynaud phenomenon, autoimmune hemolytic anemia, thrombocytopenia, lymphopenia, anticardiolipin antibodies, lupus anticoagulant, antinuclear antibodies, anti-Sm and VDRL. Autoantibodies were investigated by ELISA (anticardiolipin and anti-Sm), indirect immunofluorescency (antinuclear antibodies) and by dilute prothrombin test, coagulation Kaolin test and dilute Russell viper venom test (lupus anticoagulant). Results: Arterial thrombosis frequency was increased in PAPS $(59.3 \%$ vs $25.0 \%, \mathrm{p}=0.009)$ and venous thrombosis in SAPS (53.1\% vs $33.3 \% ; p>0.05)$, while there was no differences between frequencies of fetal loss (50.0\% vs $56.7 \%$ ), Raynaud phenomenon (18.5\% vs $18.8 \%)$, reticular livedo (18.5\% vs $12.5 \%)$, lupus anticoagulant (33.3\% vs $37.5 \%)$, IgG anticardiolipin antibodies $(79.2 \%$ vs $72,4 \%)$ and IgM anticardiolipin antibodies $(58.4 \%$ vs $65.5 \%)$ in the two groups evaluated. Moreover, an increased frequency of lymphopenia (71.2\% vs $7.4 \%$; $\mathrm{p}<0.0001)$, antinuclear antibodies (100\% vs 7.4\%; $\mathrm{p}<0.0001)$ and positive VDRL $(47.1 \%$ vs $5.0 \%, \mathrm{p}=0.005)$ was observed in SAPS. Conclusions: Clinical and laboratory features were similar on PAPS and SAPS, although arterial thrombosis was associated to PAPS, and lymphopenia, antinuclear antibodies and VDRL were associated to SAPS.
\end{abstract}

Keywords: antiphospholipid syndrome, systemic lupus erythematosus, thrombosis, anticardiolipin antibodies, lupus anticoagulant.

\footnotetext{
* Departamento de Clínica Médica. Hospital das Clínicas da Faculdade de Medicina de Ribeirão Preto (HC/FMRP/USP). Recebido em 9/10/2001. Aprovado, após revisão, em 2/10/2002.

1. Divisão de Imunologia Clínica (HC/FMRP/USP).

2. Divisão de Hematologia (HC/FMRP/USP).
}

Endereço para correspondência: Paulo Louzada Júnior. Divisão de Imunologia Clínica, Departamento de Clínica Médica, Faculdade de Medicina de Ribeirão Preto, Universidade de São Paulo, CEP 14.048.900, Ribeirão Preto, SP, Brasil, e-mail: plouzadajr@uol.com.br 


\section{INTRODUÇÃO}

A síndrome do antifosfolípide (SAF) é uma doença autoimune caracterizada pela associação de manifestações clínicas como trombose e/ou perda gestacional e a presença de anticorpos antifosfolípides. Tais anticorpos constituem um grupo de auto-anticorpos, incluindo anticardiolipina, anti- $\beta 2$-glicoproteína I ( $\beta 2-\mathrm{GPI})$ e anticoagulante lúpico, dirigidos contra complexos de proteínas plasmáticas e fosfolípides ${ }^{(1,2)}$. A SAF foi originalmente descrita em pacientes com lúpus eritematoso sistêmico (LES), porém, desde os primeiros relatos, já se reconhecia a presença das manifestações clínicas de SAF em pacientes sem LES $^{(3)}$. Esse grupo foi denominado como SAF primária ${ }^{(4)}$. Assim sendo, a SAF é classificada em SAF primária (SAFP), quando não há outras doenças associadas, e SAF secundária (SAFS), quando está associada com outras doenças ${ }^{(5)}$. A principal patologia associada com SAFS é o LES. Contudo, a SAFS pode estar associada com outras patologias auto-imunes, com neoplasias malignas, com doenças infecciosas e com o uso de drogas. Na maioria dessas condições, exceto o LES, a presença de trombose é rara ${ }^{(6)}$.

A classificação da SAF é realizada segundo os critérios estabelecidos em 1999(7) (Tabela 1).

\section{TABELA 1}

Critérios de diagnóstico de SAF SEgundo ReFErÊnCiA ${ }^{(6)}$

\section{Critérios clínicos}

1. Um ou mais episódios de trombose vascular: venosa, arterial ou de pequenos vasos.

2. Morbidade gestacional.

a. Três ou mais abortamen tos consecutivos espontâneos ( $<10$ semanas), sendo outras causas excluídas.

b. Uma ou mais mortes inexplicadas de fetos morfologicamente normais (>10 semanas).

c. Um ou mais nascimentos prematuros de crianças morfologicamente normais (<34 semanas) em conseqüên cia de préeclâmpsia grave, eclâmpsia ou insuficiência placentária.

\section{Critérios laboratoriais}

1. Anticorpo anticardiolipina, isotipo IgG ou IgM, presente no sangue em títulos moderados ou altos, em duas ou mais ocasiões, com intervalos de seis ou mais seman as e pesquisados por um ELISA padronizado para anticorpos anticar diolipina dependentes de b2-GP1.

2. Inibidor lúpico presente no plasma em duas ou mais ocasiões, com intervalos de seis ou mais seman as, detectado conforme as diretrizes da Sociedade Intern acional em Trombose e Hemostasia, Subcomitê em Inibidor Lúpico/Anticorpos An tifosfolípides.

0 paciente que apresenta um critério clínico e um critério laboratorial tem o diagnóstico de SAF confirmado.
Atualmente, a SAF é reconhecida como a principal causa de hipercoagulabilidade adquirida, bem como está intimamente relacionada com a morbidade e com a mortalidade no $\operatorname{LES}^{(8)}$.

Os pacientes com SAF apresentam trombose arterial ou venosa recorrentes, podendo afetar qualquer vaso sangüíneo. Geralmente, os fenômenos arteriais são seguidos por novos fenômenos arteriais, assim como os fenômenos venosos são seguidos por novos fenômenos venosos. A manifestação clínica mais comum na SAF é a trombose venosa profunda (TVP) em membros inferiores, acompanhada por tromboembolismo pulmonar (TEP) em 30\% dos $\operatorname{casos}^{(8)}$. A principal manifestação arterial é a isquemia cerebral, em conseqüência da trombose ou da embolia arterial no sistema nervoso central (SNC). Os episódios de isquemia cerebral podem ser permanentes ou transitórios e afetam homens e mulheres igualmente, com idade inferior a $50 \operatorname{anos}^{(9)}$. O livedo reticular e as úlceras são as manifestações cutâneas mais comuns na $\operatorname{SAF}^{(10,11)}$.

O diagnóstico de SAF requer a presença de manifestações clínicas de trombose vascular ou perda fetal recorrente, bem como a presença de alterações laboratoriais específicas e permanentes, que podem ser a detecção de anticorpos anticardiolipina (ACA) ou a determinação da presença do inibidor ou anticoagulante lúpico ${ }^{(2,7,12)}$.

O objetivo deste estudo foi traçar um perfil dos pacientes com SAF acompanhados em um serviço de referência da região nordeste do Estado de São Paulo, com ênfase nos aspectos clínicos e laboratoriais mais relevantes à doença.

\section{PACIENTES E MÉTODOS}

Avaliamos um grupo de 59 pacientes com SAF acompanhados no ambulatório de colagenoses do Hospital das Clínicas da Faculdade de Medicina de Ribeirão Preto/UsP, no período de 1996 a 2000.

O grupo foi dividido em dois subgrupos constituídos por 27 pacientes com SAFP e 32 pacientes com SAFS ao LES. O diagnóstico de SAF foi estabelecido segundo critérios definidos no VIII Simpósio Internacional em Anticorpos Antifosfolípides ${ }^{(7)}$ (Tabela 1). Os pacientes foram avaliados quanto à presença de fenômenos trombóticos arteriais e venosos, insucessos obstétricos, fenômeno de Raynaud, livedo reticular, anemia hemolítica auto-imune (AHAI), plaquetopenia, linfopenia, anticorpos anticardiolipina, inibidor lúpico, anticorpos antinucleares, anti-Sm e VDRL.

A detecção de anticorpos anticardiolipina foi realizada por ELISA "sanduíche", para o qual foram utilizados kits "Kallestad Anti-Cardiolipin Microplate EIA" adquiridos da 
Sanofi Pasteur Diagnostics (France). Os resultados foram obtidos pela leitura das absorvâncias de cada poço em um espectrofotômetro. A quantidade de anticorpos ligados foi lida em termos de unidades de absorvância. As concentrações de anticorpos anticardiolipina IgG ou IgM nas amostras foram estimadas pela interpolação das unidades de absorvância em uma curva padrão. Essa curva foi baseada em valores obtidos de um conjunto de amostras padronizadas com o Laboratório de Padronização de Antifosfolípides (Universidade de Louisville, USA). Os valores de anticorpos anticardiolipina IgG e IgM são expressos em unidades $\mathrm{GPL} / \mathrm{ml}$ e MPL/ $\mathrm{ml}$, respectivamente. $\mathrm{O}$ valor de corte definido pelo fornecedor do kit, obtido a partir da determinação de 172 amostras de indivíduos aparentemente normais, doadores de sangue, foi 10 unidades GPL $/ \mathrm{ml}$ e 10 unidades $\mathrm{MPL} / \mathrm{ml}$ para $\operatorname{IgG}$ e IgM, respectivamente. Valores entre 10 e 15 unidades foram definidos como fracamente positivos, entre 15 e 60 unidades foram definidos como moderadamente positivos e valores acima de 60 unidades como fortemente positivos.

A presença do inibidor lúpico foi determinada por intermédio de três testes diferentes. Assim, realizava-se primeiro o teste do tempo de protrombina diluída (TPd), seguido do tempo de coagulação do caulin (TCK) e, por fim, o tempo do veneno de víbora de Russell diluído (dRVVT). Se o paciente apresentasse positividade em algum dos testes, ficaria suspensa a realização dos testes subseqüentes, por ter sido demonstrada a presença do inibidor lúpico. Tanto a detecção dos anticorpos anticardiolipina como a determinação do inibidor lúpico foram realizadas em duas ocasiões, dentro de um intervalo de tempo mínimo de seis semanas.

Para determinação do fator antinúcleo (FAN) utilizamos a técnica de imunofluorescência indireta, tendo como substrato núcleos de fígado de camundongo e de células HEp2. A determinação dos anticorpos anti-Sm foi realizada por um imunoensaio enzimático semiquantitativo utilizando kits Sigma-Chemical (Saint Louis, MO, USA).

Para as análises comparativas dos resultados obtidos utilizamos o teste exato de Fisher bicaudal, sendo considerados significantes os valores de $p$ menores do que 0,05 .

\section{RESULTADOS}

Dentre os pacientes com SAFP, 18 (66,7\%) eram do sexo feminino e $9(33,3 \%)$ do masculino, com idade variando entre 3 e 64 (média $=37,6)$, sendo $20(74,1 \%)$ caucasianos, $4(14,8 \%)$ pardos e $3(11,1 \%)$ negros. Dentre os pacientes com SAFS a LES, $30(93,8 \%)$ eram do sexo feminino e 2 $(6,2 \%)$ do masculino, com idades variando entre 13 e 63 (média $=36,1)$, sendo $26(81,2 \%)$ caucasianos, $3(9,4 \%)$ pardos e $3(9,4 \%)$ negros.

No grupo de pacientes com SAFP, observamos que 59,3\% $(16 / 27)$ apresentaram trombose arterial, 33,3\% (9/27) trombose venosa, 50,0\% (9/18) perda fetal, 33,3\% (9/27) inibidor lúpico presente e $66,7 \%(18 / 27)$ anticorpo anticardiolipina em títulos moderados a altos. Por outro lado, no grupo de pacientes com SAFS, 25,0\% (8/32) apresentaram trombose arterial, 53,1\% (17/32) trombose venosa, 56,7\% $(17 / 30)$ perda fetal, 37,5\% (12/32) inibidor lúpico presente e $68,8 \%(22 / 32)$ anticorpo anticardiolipina em títulos moderados a altos. Comparativamente, a freqüência de trombose arterial foi maior no grupo de pacientes com SAFP do que no grupo com SAFS $(p=0,009)$, enquanto observamos o inverso com relação à ocorrência de trombose venosa, porém, sem significância estatística (Tabela 2).

O principal tipo de trombose nos pacientes com SAFP foi a trombose em SNC $(59,3 \%)$, seguida de TEP $(29,6 \%)$, TVP $(22,2 \%)$ e, por fim, trombose visceral $(3,7 \%)$. Com relação aos pacientes comSAFS, observamos TVP em 37,5\%, seguida de trombose visceral e em SNC em 18,8\% e, por fim, TEP em 12,5\% (Tabela 3).

TABELA 2

FREQÜÊNCIA DOS CRITÉRIOS DIAGNÓSTICOS EM PACIENTES COM SAFP $(\mathrm{N}=27)$ E COM SAFS $(\mathrm{N}=32)$

\begin{tabular}{lcc}
\hline Critérios diagnósticos & $\begin{array}{c}\text { SAFP } \\
\mathbf{n}(\%)\end{array}$ & $\begin{array}{c}\text { SAFS } \\
\mathbf{n}(\%)\end{array}$ \\
\hline Trombose arterial & $16(59,3)^{\mathrm{a}}$ & $8(25,0)^{\mathrm{a}}$ \\
Trombose venosa & $9(33,3)^{\mathrm{b}}$ & $17(53,1)^{\mathrm{b}}$ \\
Insucesso obstétrico & $9(50,0)^{\mathrm{b}}$ & $17(56,7)^{\mathrm{b}}$ \\
Inibidor lúpico & $9(33,3)^{\mathrm{b}}$ & $12(37,5)^{\mathrm{b}}$ \\
Anticorpo anti cardiolipina & & \\
IgG ou IgM>15 UGPL/UMPL/mL & $18(66,7)^{\mathrm{b}}$ & $22(68,8)^{\mathrm{b}}$ \\
\hline
\end{tabular}

TABELA 3

CARACTERIZAÇÃO DOS PACIENTES COM SAFP $(\mathrm{N}=27)$ E SAFS $(\mathrm{N}=32)$ SEGUNDO A LOCALIZAÇÃO DA TROMBOSE

\begin{tabular}{lcc}
\hline Localização da trombose & SAFP & SAFS \\
& $\mathbf{n}(\%)$ & $\mathbf{n}(\%)$ \\
\hline TVP & $6(22,2)$ & $12(37,5)$ \\
TEP & $8(29,6)$ & $4(12,5)$ \\
VisC & $1(3,7)$ & $6(18,8)$ \\
SNC & $16(59,3)$ & $6(18,8)$ \\
\hline
\end{tabular}

TVP: trombose venosa profunda; TEP: tromboembolism o pulmonar; Visc: trombose visceral; SNC: trombose em sistema nervoso central 
A perda gestacional foi observada em 50,0\% das mulheres com SAFP e em $56,7 \%$ das mulheres com SAFS a LES (Tabela 2), sendo o segundo trimestre o principal período de ocorrência de perda gestacional nos dois grupos estudados, $66,7 \%$ e $64,7 \%$, respectivamente.

A Tabela 4 demonstra as frequiências dos valores de anticorpos anticardiolipina em pacientes com SAFP e com SAFS ao LES, respectivamente. Observamos uma freqüência de $79,2 \%$ de positividade para o anticorpo anticardiolipina IgG e $58,4 \%$ para o IgM, em pacientes com SAFP. Enquanto nos pacientes com SAFS, observamos uma freqüência de $72,4 \%$ de positividade para o anticorpo IgG e $65,5 \%$ para o IgM. Agrupando os resultados por faixas de valores, observamos que $45,9 \%$ dos pacientes com SAFP tiveram ACA IgG em títulos moderados a altos, enquanto 41,7\% destes pacientes tiveram ACA IgM nestes títulos. Apesar de a presença do ACA subtipo IgM poder estar associada mais comumente com infecções, todos os pacientes do estudo foram avaliados com relação à presença de infecções virais e bacterianas subjacentes, cujo resultado foi negativo. Além disso, quatro indivíduos que apresentaram trombose com ACA-IgM positivo fizeram troca de positividade para IgG (negativando o ACA-IgM), após um ano do diagnóstico inicial. Com relação aos pacientes com SAFS, 51,7\% apresentaram ACA IgG em títulos moderados a altos e $58,6 \%$ tiveram o subtipo IgM nestes títulos.

TABELA 4

FreqüÊNCIA DE ACA IGG E IGM, POR FAIXAS DE VALORES, EM PACIENTES COM SAFP $(\mathrm{N}=24)$ E NAQUELES COM SAFS AOLES $(\mathrm{N}=29)$

\begin{tabular}{|c|c|c|c|c|c|}
\hline \multicolumn{6}{|c|}{ SAFP } \\
\hline $\begin{array}{l}\text { ACA - IgG } \\
\text { (UGPL/ml) }\end{array}$ & $n$ & $\%$ & $\begin{array}{l}\text { ACA - IgM } \\
\text { (UMPL/ml) }\end{array}$ & n & $\%$ \\
\hline$<\mathrm{ou}=10$ & 5 & 20,8 & $<\mathrm{ou}=10$ & 10 & 41,7 \\
\hline $11-15$ & 8 & 33,3 & $11-15$ & 4 & 16,7 \\
\hline $16-60$ & 4 & 16,7 & $16-60$ & 9 & 37,5 \\
\hline$>60$ & 7 & 29,2 & $>60$ & 1 & 4,2 \\
\hline \multicolumn{6}{|c|}{ SAFS } \\
\hline $\begin{array}{l}\text { ACA - IgG } \\
\text { (UGPL/ml) }\end{array}$ & $\mathbf{n}$ & $\%$ & $\begin{array}{l}\text { ACA - IgM } \\
\text { (UMPL/ml) }\end{array}$ & $n$ & $\%$ \\
\hline$<\mathrm{ou}=10$ & 8 & 27,6 & $<\mathrm{ou}=10$ & 10 & 34,5 \\
\hline $11-15$ & 6 & 20,7 & $11-15$ & 2 & 6,9 \\
\hline $16-60$ & 8 & 27,6 & $16-60$ & 14 & 48,3 \\
\hline$>60$ & 7 & 24,1 & $>60$ & 3 & 10,3 \\
\hline
\end{tabular}

A avaliação dos pacientes quanto à freqüência de outras manifestações clínicas e laboratoriais, não incluídas entre os critérios de diagnóstico de SAF, revelou uma freqüência aumentada de AHAI $(25,0 \%$ vs $7,4 \%, p>0,05)$, plaquetopenia $(40,6 \%$ vs $22,2 \%, p>0,05)$, linfopenia $(71,2 \%$ vs $7,4 \%$, $p<0,0001)$, FAN positivo ( $100 \%$ vs $7,4 \%, p<0,0001)$, anticorpos anti-Sm positivos $(18,8 \%$ vs $0 \%, p=0,07)$ e VDRL positivo $(47,1 \%$ vs $5,0 \%, p=0,005)$ na SAFS com relação aos pacientes com SAFP (Tabela 5).

TABELA 5

OUTRAS MANIFESTAÇÕES CLÍNICAS E LABORATORIAIS EM PACIENTES COM SAFP $(\mathrm{N}=27)$ E COM SAFS $(\mathrm{N}=32)$

\begin{tabular}{lcc}
\hline Manifestações clínicas & $\begin{array}{c}\text { SAFP } \\
\mathbf{n ~ ( \% )}\end{array}$ & $\begin{array}{c}\text { SAFS } \\
\mathbf{n}(\%)\end{array}$ \\
\hline Raynaud & $5 / 27(18,5)^{\mathrm{a}}$ & $6 / 32(18,8)^{\mathrm{a}}$ \\
\hline Livedo & $5 / 27(18,5)^{\mathrm{a}}$ & $4 / 32(12,5)^{\mathrm{a}}$ \\
AHAI & $2 / 27(7,4)^{\mathrm{a}}$ & $8 / 32(25,0)^{\mathrm{a}}$ \\
\hline Manifestações laboratoriais & SAFP & SAFS \\
& $\mathrm{n}(\%)$ & $\mathrm{n}(\%)$ \\
\hline Plaquetopenia & $6 / 27(22,2)^{\mathrm{a}}$ & $13 / 32(40,6)^{\mathrm{a}}$ \\
\hline Linfopenia & $2 / 27(7,40)^{\mathrm{b}}$ & $23 / 32(71,2)^{\mathrm{b}}$ \\
Anti-Sm + & 0 & $6 / 32(18,8)$ \\
\hline Anti-Sm - & $20 / 20(100)^{\mathrm{a}}$ & $26 / 32(81,3)^{\mathrm{a}}$ \\
\hline FAN + & $2 / 27(7,4)^{\mathrm{b}}$ & $32 / 32(100)^{\mathrm{b}}$ \\
\hline FAN - & $25 / 27(92,6)$ & 0 \\
VDRL + & $1 / 20(5,0)^{\mathrm{c}}$ & $8 / 17(47,1)^{\mathrm{c}}$ \\
\hline VDRL - & $19 / 20(95,0)$ & $9 / 17(52,9)$ \\
\hline
\end{tabular}

AHAI: Anemi a hem olítica auto-imune; FAN: Fator an tinúcleo; VDRL: “Veneral Disease Reference Laboratory"; $\mathrm{a}-p>0,05 ; \mathrm{b}-p<0,0001 ; \mathrm{c}-p=0,005$

\section{DISCUSSÃO}

A SAF ainda é uma doença pouco documentada no Brasil, sendo poucos os estudos em populações brasileiras ${ }^{(13,14,15)}$. Neste estudo, objetivamos traçar um perfil clínico e laboratorial dos pacientes com SAF acompanhados em um hospital universitário da região nordeste do Estado de São Paulo. A trombose arterial foi mais freqüente nos pacientes com SAFP que naqueles com SAFS ao LES $(p=0,009)$, enquanto ocorreu o inverso com a freqüência de evento trombótico em sítio venoso, porém, sem significância estatística (Tabela 2). Em um estudo comparativo com 58 pacientes com SAFP e 56 com SAFS ao LES, mostrou-se 
que não há diferenças estatísticas entre os dois grupos quanto à frequiência de manifestações trombóticas, sendo que a trombose venosa foi mais freqüente em ambos os grupos: SAFP e SAFS ao $\mathrm{LES}^{(10)}$. É possível que a freqüência aumentada de trombose arterial nos pacientes com SAFP que estudamos seja apenas um achado ocasional e relacionado com o tipo de paciente que recorre à assistência em nível terciário, pois as tromboses arteriais são mais incomuns do que as tromboses venosas na população geral. Além disso, os pacientes jovens com trombose arterial são encaminhados para investigação em nível terciário mais precocemente, enquanto os indivíduos com trombose venosa, em geral, são tratados em nível secundário sem uma investigação mais detalhada para possíveis causas de trombofilia, exceto quando apresentam fenômenos trombóticos repetidos. Em nosso estudo, o principal tipo de trombose arterial observado no grupo de pacientes com SAFP foi a de SNC (59,2\%), enquanto TVP foi observada em apenas 22,2\% dos pacientes (Tabela 3). Tal observação explica-se pela alta freqüência de fenômenos trombóticos arteriais observada neste grupo de pacientes, sendo a isquemia cerebral a principal manifestação de trombose arterial nos pacientes com $\mathrm{SAF}^{(10)}$. A principal manifestação trombótica no grupo com SAFS foi a TVP, em 37,5\% dos casos, sendo esta a manifestação trombótica mais comumente encontrada nos pacientes com $\mathrm{SAF}^{(9)}$. Cabe ressaltar a presença de uma paciente com três anos de idade, com quadro de acidente vascular cerebral aos 18 meses de vida (artéria cerebral posterior e basilar), confirmado por ressonância nuclear magnética (áreas de infarto no putâmen e núcleo caudado à esquerda) e anticorpo anticardiolipina positivo em títulos elevados. Não possuía história familiar de trombose. Os auto-anticorpos foram todos negativos e o ecocardiograma normal. Apesar de não haver distinção clínica entre a SAF em crianças como em adultos, o número de casos é bem menor. Além disso, a ocorrência em crianças menores de dois anos é bastante rara ${ }^{(16,17)}$. Porém, o reumatologista deve atentar para a possibilidade da ocorrência de SAF inclusive em crianças.

Com relação aos insucessos obstétricos, tanto na SAFP quanto na SAFS observamos a perda fetal no segundo trimestre de gestação como principal evento $(66,7 \%$ e $64,7 \%$, respectivamente), não havendo diferenças estatísticas entre os dois grupos estudados. Os anticorpos antifosfolípides estão associados com uma grande variedade de insucessos obstétricos, como abortamentos recorrentes, perdas fetais no segundo trimestre de gestação, pré-eclâmpsia, retardo de crescimento intra-uterino e nascimentos prematuros ${ }^{118}$ 19). A incidência de perda gestacional, em qualquer período da gestação, em pacientes com anticorpos antifosfolípides, foi de $47 \%$, enquanto a incidência de abortamentos espontâneos e a de perda fetal foi de $20 \%$, cada ${ }^{(20)}$. Os achados histopatológicos típicos em placentas de mães com SAF são trombose placentária e infarto. Entretanto, em muitos casos de perda gestacional, especialmente em abortamento espontâneo, não há evidências de trombose placentária, podendo haver outros mecanismos envolvidos na perda gestacional da SAF, como: alterações na secreção placentária de gonadotrofina coriônica humana ${ }^{(21)}$, reatividade cruzada dos anticorpos antifosfolípides com fosfolípides trofoblásticos e conseqüente exposição das vilosidades trofoblásticas à destruição por células maternas imunoefetoras ${ }^{(22)}$ e redução dos níveis séricos de interleucina-3 induzida pelo anticorpo anticardiolipina ${ }^{(23)}$.

A distribuição de anticorpos anticardiolipina mostrou uma freqüência de $79,2 \%$ de positividade para o anticorpo anticardiolipina IgG e $58,4 \%$ para o IgM em pacientes com SAFP. Com relação aos pacientes com SAFS ao LES, 72,4\% apresentaram anticorpo anticardiolipina IgG positivo e $65,5 \%$ apresentaram IgM positivo. Agrupando os resultados por faixas de valores, observamos que $45,9 \%$ dos pacientes com SAFP tiveram anticorpo anticardiolipina $\operatorname{IgG}$ em títulos moderados a altos, enquanto $41,7 \%$ destes pacientes tiveram IgM nestes títulos. Com relação aos pacientes com SAFS ao LES, observamos que $51,7 \%$ tiveram anticardiolipina IgG em títulos moderados a altos e 58,6\% tiveram o subtipo IgM nestes títulos (Tabela 4). A distribuição de anticorpos anticardiolipina em pacientes com SAF, habitualmente, é semelhante entre os pacientes com SAFP e aqueles com SAFS ao LES, sendo a freqüência do subtipo IgM menor que a freqüência do subtipo IgG, conforme mostrado previamente ${ }^{(10)}$. No entanto, em um seguimento de dez anos, ficou demonstrado que pacientes com SAFS a LES apresentam uma persistência prolongada dos títulos de anticorpo anticardiolipina $\operatorname{IgM}^{(24)}$. Em nosso estudo, observamos uma tendência de aumento da freqüência de anticorpo anticardiolipina IgM com relação ao subtipo $\operatorname{IgG}(58,6 \%$ e $51,7 \%$, respectivamente) no subgrupo de pacientes com SAFS a LES. Contudo, essa diferença não foi estatisticamente significante. O subtipo IgM do anticorpo anticardiolipina tem sido associado com fenômenos trombóticos em sítios venosos e não arteriais ${ }^{(25)}$, justificando, em parte, uma maior incidência de trombose venosa que trombose arterial $(53,1 \%$ e $25,0 \%$, respectivamente) nos pacientes com SAFS a LES avaliados neste estudo. 
Com relação ao inibidor ou anticoagulante lúpico, não houve diferença entre os pacientes com SAFP e SAFS (33,3\% vs. $37,5 \%$, respectivamente). O termo anticoagulante lúpico foi introduzido em $1977^{(26)}$ e, por definição, o anticoagulante lúpico é uma mistura de imunoglobulinas (IgG, $\operatorname{IgM}$ e $\operatorname{IgA}$ ) que interferem com um ou mais testes da coagulação dependentes de fosfolipídio (TTPa, TPd, TCK ou dRVVT). Esses anticorpos, apesar de definidos como antifosfolípides, reconhecem, na verdade, várias proteínas plasmáticas que se ligam a fosfolipídios como, por exemplo, a B2-GPI, a protrombina, a anexina $\mathrm{V}$, o cininogênio de alto peso molecular e o fator $\mathrm{XI}^{(2)}$. A protrombina e a B2-GPI são os alvos mais comuns dos anticorpos antifosfolípides. Desta forma, variando com o tipo de teste utilizado para a pesquisa do anticoagulante lúpico, a detecção destes anticorpos torna-se possível ou não. Exemplificando: o TCK é mais sensível para a detecção de anticorpos antiprotrombina, enquanto o dRVVT é mais sensível para o anti-B2-GPI ${ }^{(27)}$. A realização dos três métodos supracitados para a identificação do anticoagulante lúpico parece ser mais apropriada, pois possibilita detectar os vários subtipos de anticorpos contra proteínas plasmáticas ligantes de fosfolipídios.

Dentre as outras manifestações da SAF, observamos que a frequiência de fenômeno de Raynaud, livedo reticular, anemia hemolítica autoimune, plaquetopenia e anticorpo anti-Sm foi estatisticamente semelhante entre os dois

\section{REFERÊNCIAS}

1. Hughes GRV: The antiphospholipid syndrome. Lupus 5:345-6, 1996.

2. Triplett DA: Use of the dilute Russell viper venom time (dRVVT): its importance and pitfalls. J Autoimmun, 15:173-8, 2000.

3. Hughes GRV: Thrombosis, abortion, cerebral disease and the lupus anticoagulant. Br Med J 287:1088-89, 1983.

4. Asherson RA: A "primary" antiphospholipid syndrome? J Rheumatol 15:1742-46, 1988.

5. Asherson RA, Cervera R: "Primary", "secondary" and others variants of the antiphospholipid syndrome. Lupus 3:293-8, 1994.

6. Asherson RA, Cervera R, Piette JC, Shoenfeld Y: The Antiphospholipid Syndrome: History, definition, Classification and Differential Diagnosis. In: Asherson RA, Cervera R, Piette JC, Shoenfeld Y (eds), The Antiphospholipid Syndrome, 1 st ed, Florida, CRC Press, 3-12, 1996.

7. Wilson WA, Gharavi AE, Koike T, Lockshin MD, Branch DW, Piette JC: International consensus statement on preliminary classification criteria for definite antiphospholipid syndrome: report of an international workshop. Arthritis Rheum 42:1309-11, 1999.

8. Petri M: Epidemiology of the antiphospholipid antibody syndrome. J Autoimmun 15:45-151, 2000. grupos estudados, apesar de observarmos a tendência do aumento na freqüência de anemia hemolítica autoimune, plaquetopenia e anti-Sm na SAFS ao LES. Foram descritas associações entre anticorpos antifosfolípides e a ocorrência de plaquetopenia e de anemia hemolítica em pacientes com SAFP e SAFS ao LES. Contudo, a anemia hemolítica parece estar mais associada com SAFS ao LES ${ }^{(9)}$. Ademais, observamos a freqüência aumentada de linfopenia e anticorpos antinucleares na SAFS ao LES (Tabela 5). A presença de anticorpos antinucleares demonstrados por imunofluorescência indireta é descrita em pacientes com SAFP e SAFS ao LES, porém, quando presentes em pacientes com SAFP, estão em títulos baixos, 1:40 a 1:160. Anticorpos contra antígenos nucleares extraíveis estão ausentes na SAFP e podem ser encontrados na SAFS ao LES $^{(4)}$. Curiosamente, observamos diferença entre as freqüências de VDRL positivo nos dois subgrupos, 5,0\% no subgrupo com SAFP e $47,1 \%$ no com SAFS ao LES (Tabela 5), dado este sem relatos prévios na literatura.

Em conclusão, as manifestações clínicas na SAFP e na SAFS ao LES foram semelhantes. Contudo, a trombose arterial foi mais freqüente na SAFP, sendo a isquemia cerebral a principal manifestação trombótica nestes pacientes, enquanto a TVP foi a principal manifestação trombótica na SAFS ao LES. Assim como a linfopenia, a presença de anticorpos antinucleares e o VDRL positivo foram mais freqüentes na SAFS ao LES.

9. Amigo MC, Khamashta MA: Antiphospholipid (Hughes) syndrome in systemic lupus erythematosus. Rheum Dis Clin Nor Am 26:331-48, 2000.

10. Ordi-Ros J, Selva-O'Callaghan, Vilardell-Tarrés. Thrombotic manifestations in Antiphospholipid Syndrome. In: Asherson RA, Cervera R, Piette JC, Shoenfeld Y (eds), The Antiphospholipid Syndrome, $1^{\text {st }}$ ed, Florida, CRC Press, 107-16, 1996.

11. Vianna JL, Khamashta MA, Ordi-Ros J, et al: Comparison of the primary and secondary antiphospholipid syndrome: a European multicenter study of 114 patients. Am J Med 96:3-9, 1994.

12. Carreras LO, Farastiero RR, Martinuzzo ME: Which are the best biological markers of the antiphospholipid syndrome? J Autoimmun 15:163-72, 2000.

13. Dahmer R, Staub HL: Síndrome antifosfolipídica: os dilemas dos novos critérios. Rev Bras Reumatol 41:59-61, 2001.

14. Couto E, Barini R, Silva JLC, Moraes DRLP, Carvalho LMF: Anticardiolipin antibody in recurrent spontaneous aborting and fertile women. Rev Paul Med 116(4): 1760-65, 1998.

15. Celli CM, Gharavi AE: Origin and pathogenesis of antiphospholipid antibodies. Braz J Med Biol Res 31(6):723-32, 1998.

16. Sammaritano LR: Pediatric and Familial Antiphospholipid Syndromes. In: Asherson RA, Cervera R, Piette JC, Shoenfeld Y (eds), The Antiphospholipid Syndrome,. $1^{\text {st }}$ ed, Florida, CRC Press, 25966, 1996. 
17. Roddy SM, Giang DW: Antiphospholipid antibodies and stroke in an infant. Pediatrics 87:933-5, 1991.

18. Scott RAH: Anti-cardiolipin antibodies and preeclampsia. Br J Obstet Gynecol 94:604-5, 1987.

19. Rai R: Obstetric management of antiphospholipid syndrome. J Autoimmun 15:203-7, 2000.

20. Triplett DA: Antiphospholipid antibodies and recurrent pregnancy loss. Am J Reprod Immunol 20(2):52-67, 1989.

21. Shurtz-Swirski R, Inbar O, Blank J, et al : In vitro effect of anticardiolipin autoantibodies upon total and pulsatile placental HCG secretion during early pregnancy. Am J Reprod Immunol 29:206-10, 1993.

22. McCrae KR, DeMichele AM, Pandhi P, et al: Detection of antitrophoblast antibodies in the sera of patients with anticardiolipin antibodies and fetal loss. Blood 82(9): 2730-41, 1993.
23. Louzada Jr P, Simon MS, Voltarelli JC, Donadi EA: Síndrome do anticorpo antifosfolípide. Medicina 31:305-15, 1998.

24. Alarcón-Segovia D, Pérez-Ruiz A, Villa AR: Log-term prognosis of antiphospholipid syndrome in patients with systemic lupus erythematosus. J Autoimmun 15:157-61, 2000.

25. Horbach DA, Oort EV, Donders RCJM, Derksen RHWM, De Groot PG: Lupus anticoagulant is the strogest risk factor for both venous and arterial thrombosis in patients with systemic lupus erythematosus. Thromb Haemost 76(6):916-24, 1996.

26. Feinstein DI, Rapaport SI: Acquired inhibitors of blood coagulation. Prog Haemostas Thromb 1:75-95, 1977.

27. Galli M, Barbui T: Antiprothrombin antibodies: detection and clinical significance in the antiphospholipid syndrome. Blood 93:2149-57, 1999. 